\title{
DIFERENCIAS DE GÉNERO EN LA FUNCIÓN TUTORIAL
}

\author{
María González Álvarez \\ Consejería de Educación del Principado de Asturias
}

RESUMEN: La función tutorial se reconoce en la literatura como un componente básico de la práctica docente y factor de calidad de la enseñanza. En el presente trabajo pretendemos analizar, en función del género, distintos aspectos de la tutoría de los centros de Educación Infantil, Educación Primaria, Educación Secundaria Obligatoria, Bachillerato y Formación Profesional. Con este fin hemos elaborado una prueba específica que ha sido enviada por correo electrónico a una muestra de tutores y tutoras de todas las comunidades de España y que ha sido cumplimentada por 1350. Hemos realizado un trabajo exploratorio para obtener información sobre la actual situación de la función tutorial respecto a diversos aspectos. Las tutoras valoran mejor que los tutores la formación inicial y permanente recibida, así como la importancia que tienen diversos temas para su formación. También valoran mejor las tutoras otros aspectos como la importancia que tiene la tutoría para la educación del alumnado y para el funcionamiento del centro, el reconocimiento y colaboración que tienen por parte de administraciones educativas, profesorado y familias, así como determinadas mejoras profesionales que deberían realizarse. Podría analizarse si este mejor perfil de las docentes tutoras puede suponer una mejora en la calidad de la enseñanza.

PALABRAS CLAVE: Género, función tutorial, formación permanente, satisfacción laboral.

\section{GENDER DIFFERENCES IN THE TUTORING FUNCTION}

ABSTRACT: Head teacher's roles are accepted in the specialized literatura as a basic component of the teaching practice and a main factor in achieving the teaching quality. In this paper we try to analize the different head teachers'tasks in Preeschool, Primary, Secondary, Baccalaureate and Vocational Training Education. For that purpose we have written a specific test that has been emaliled to a simple of head teachers from all the different comunities of Sapain and has been filled in by 1350 head teachers. We have cinouted an exploratory study that has provided 
us whit information about the current situation of the head teachers' takes domen head teachers value more that men initial and ongoing training as well as the importance of certain topies related to their training. They also vaue better other aspects such as the importance of the head teachers'tasks for the students'education and the running of the eschool, the recognition and collaboration from the educational autorities, teachers and families and some propessiond improvaments that they considered as necessary. It could be analyzed if this better profile of the head teachers women can influence an improvement in the quality of teaching.

KEYWORDS: Gender; head teacher's roles, continuous training, work satisfaction.

Recibido: 29/11/2017

Aceptado: 15/01/2018

Correspondencia: María González Álvarez, Consejería de Educación, C/ Rosal, 9, 33009 Oviedo. Email: maria.gz.az@gmail.com.

\section{INTRODUCCIÓN}

La tutoría es un componente básico de la práctica docente y factor de calidad de la enseñanza (Álvarez, 2017; Cañas, Campy y Pantoja, 2005; López, 2013). Para Álvarez, "la realidad socio-educativa se encuentra en constantes cambios (...) lo cual requiere, asimismo, un cambio en los roles y funciones de la orientación y la tutoría" (2017: 2829). Por estos motivos entendemos que, después de haberse regulado por las administraciones educativas la función tutorial y la orientación, procede analizar su situación actual en las enseñanzas no universitarias en España. Para ello hemos realizado un estudio exploratorio de determinados aspectos de dicha función y, en concreto, las posibles diferencias que pudieran observarse en función del género. Hemos solicitado a tutores y tutoras sus opiniones y valoraciones respecto a sus tareas ejercidas en centros de Educación Infantil, Educación Primaria, Educación Secundaria Obligatoria (en adelante ESO), Bachillerato y FP del conjunto de las comunidades autónomas de España.

En este estudio tenemos en cuenta diversas variables tales como la edad, el tiempo de experiencia docente, tiempo de experiencia como tutor/a, titularidad del centro, número de alumnado del centro y horas semanales dedicadas a la tutoría. Aspecto fundamental a analizar en este trabajo es el referido a la formación inicial y permanente de tutores/as sobre la función tutorial. Consideramos, asimismo, la satisfacción que señalan tener en su actividad tutorial y la importancia que según su opinión tiene la tutoría en la educación del alumnado y en el funcionamiento del centro. Analizamos el reconocimiento que reciben por parte de las administraciones educativas, profesorado y familias del centro sobre su trabajo en la tutoría. Atendemos a la información que nos otorgan tutores/as respecto a que se tenga en cuenta en su carrera profesional el ejercicio de dicha función, así como la retribución económica que pudiera corresponderles. Es importante conocer las valoraciones que tutores/as realizan respecto a la colabora- 
ción que tienen en su trabajo por parte del equipo docente, familias y equipo directivo del centro. Otro aspecto de fundamental interés es la valoración que realizan respecto a la atención y dedicación que como tutores o tutoras dedican a sus tareas tutoriales.

La función tutorial debe ser un proceso continuo y sistemático de la acción educativa y que facilite el proceso de aprendizaje y alcance el desarrollo personal del alumnado (Álvarez, 2017; Ceballos, 2017), acción tutorial que debe ser colectiva y coordinada de todo el profesorado, lo que supone que debe realizarse un nuevo enfoque más comprensivo e integral de la tutoría (Álvarez 2017; Mateo 2008; Rodríguez y Romero 2015). Según Valenzuela y Sales (2016) tutores y tutoras coordinan el trabajo del equipo docente favoreciendo una practica educativa coherente y un enfoque globalizador de la acción educativa. Para González-Palacios y Avelino-Rubio (2016) debe dejarse de hablar en singular de tutoría para referirnos a tutorías.

En el análisis del desarrollo de la tutoría observa Álvarez-Justel (2015) que no ha estado totalmente integrada en la formación, careciendo en muchas ocasiones de objetivos y contenidos concretos, siendo insuficiente la disponibilidad del horario del profesorado y del alumnado para la misma y que en demasiadas ocasiones se reduce a un servicio burocrático.

Santana y Feliciano (2006) analizan la actitud y competencia de tutor y tutora, entendiendo que su tarea no es mecánica ni cualquier profesor o profesora pueden llevarla a cabo. Para Bolívar (2007) debe propiciarse una auténtica profesionalización del docente que debe conducir a un profesional del aprendizaje al tiempo que un educador.

El futuro de la acción tutorial es para Quintanal (2006) esperanzador puesto que existen bases fundadas que hacen considerar que la función tutorial jugará un papel fundamental en el desarrollo de la actividad educativa.

Respecto a la formación del profesorado en la función tutorial, y refiriéndose tanto a la inicial como a la permanente, Esteve (2007) considera que el éxito como profesor se convierte en algo que es posible objetivar, enseñar y aprender. Cañas, Campoy y Pantoja (2005) consideran necesaria una mayor formación en técnicas tutoriales, así como más recursos para llevarlas a cabo y mayor coordinación entre los tutores. Según Rodríguez y Romero (2015) aunque la formación de tutores/as no es requisito suficiente para garantizar una formación integral del alumnado, sí resulta imprescindible. Entienden, asimismo, que las propuestas de mejora deben dirigirse a prestar más atención a la formación del profesorado como tutor/a en los actuales planes de estudio universitarios, debiendo propiciarse un mayor y mejor reconocimiento de la acción tutorial. Para la profesora Martín-Moreno (2008) en dichos programas de formación tutorial deben incluirse habilidades y actitudes en relación con la igualdad de género.

En relación con la satisfacción del tutor/a en su tarea, Serrano (2009) considera que ésta es buena, aunque se encuentran decepcionados con el escaso reconocimiento que se hace de su trabajo y con su retribución económica y posibilidades de desarrollo profesional. También López (2013) señala que aunque los tutores/as se consideran preparados para ejercer su labor como tal no se sienten apoyados en ella ni su trabajo recompensado. Según Torres (2010) el profesorado que tutoriza en educación secundaria se encuentra 
bastante satisfecho con el desarrollo de su profesión docente aunque no se muestra satisfecho en sus relaciones con la administración educativa. La satisfacción laboral para Santos, Godás, Lorenzo y Gómez-Fraguela (2010) podría conformar decisivamente las actitudes de los docentes hacia la escuela, afectando positivamente a su motivación y desempeño. Para Gil-Flores (2017) la variable sexo constituye un buen predictor de la satisfacción laboral, siendo mejor la de las profesoras. Las profesoras se sienten laboralmente más satisfechas que sus colegas varones, están más a gusto con su trabajo actual y les gusta su vida en el centro docente más que a ellos y si tuvieran la oportunidad de acogerse a una jubilación voluntaria anticipada o de cambiar su actual trabajo como profesora por otro tipo de trabajo de similar estatus y categoría lo harían con una probabilidad inferior a la expresada por sus compañeros (Anaya y López, 2015).

Debemos señalar que aunque se encuentran numerosos e importantes trabajos en relación con la diferencias de género entre el profesorado (Anaya y López, 2015; Bermejo, Florencio, Ruiz y Rabazo, 2014; Gil-Flores, 2017; Sanz, Hernando y Mula, 2015; Torres, 2010), no ocurre lo mismo cuando estamos haciendo referencia a la función tutorial, no obstante entendemos que en alguna medida se pueden transferir los resultados de dichos trabajos a lo relativo a dicha función.

Para Castro, Expósito, López y Navarro (2015) tutores y tutoras constituyen un elemento fundamental para facilitar la colaboración, participación e implicación de las familias en la vida del centro. Reparaz y Jiménez (2015) consideran que se da una buena comunicación de la familia con el centro, siendo ésta satisfactoria para ambas partes. Sin embargo, a pesar de mostrar una actitud positiva hacia las tutorías, las familias destacan su falta de participación para establecer una educación compartida con los educandos en el centro (Hernández, Viudez y Guerrero, 2015). Por otro lado, según Torres (2010) el grado de aceptación que el profesorado tiene por parte de sus alumnos y compañeros es elevado.

Estudios recientes muestran que la satisfacción laboral del profesorado español disminuyó en la última década, y que resultó significativamente más baja en educación secundaria que en etapas educativas previas (Gil-Flores, 2017). No obstante, El profesorado de educación secundaria muestra, a nivel global, un grado de satisfacción catalogable como medio (Anaya y López, 2015).

\section{Método}

\section{Muestra}

Para analizar la actual situación de la función tutorial en las enseñanzas no universitarias, dada su importancia en la calidad de la enseñanza y para adecuarla a las siempre cambiantes realidades sociales en general y educativos en particular, hemos consultado las opiniones que tienen sobre su formación, funciones y otros aspectos de la función tutorial, según el género, los tutores y las tutoras de los centros de Educación Infantil, Educación Primaria, ESO, Bachillerato y FP. Con estos objetivos hemos elaborado una prueba específica que fue enviada por correo electrónico a una muestra de tutores/as de todas las comunidades autónomas de España, seleccionada 
al azar en función de la población de la provincia del centro en el que desarrolla su tarea tutorial y de la titularidad del mismo.

Dicha prueba fue cumplimentada por 1350 tutores y tutoras, en proporción a la población de su comunidad y titularidad de los centros en los que ejercen sus funciones tutoriales. El 64.9\% son tutoras y el 35.1\% tutores. Su edad media es de 46.3 años (tutoras 45.5 y tutores 47.8, grado de significación 0.000, en adelante gs). Su experiencia profesional docente media es de 19.2 años y como tutor o tutora 12.5 años. El 81.6\% ejercen su tutoría en centros públicos, el $17.4 \%$ en privados-concertados y el $1.0 \%$ en privados. Dedican una media semanal de 4.9 horas a su tarea tutorial. Según la enseñanza y/o etapa, el 5.0\% son tutores/as de Educación Infantil, 23.5\% de Educación Primaria, 52.1\% ESO, 9.0\% Bachillerato y el $10.0 \%$ FP. Los tutores/as que responden a nuestro protocolo ejercen sus funciones en centros con una media de 538 alumnos/as, con un total de 727.265 alumnos/as.

Tabla 1. Características personales y profesionales de tutores y tutoras

\begin{tabular}{|c|c|c|c|c|}
\hline \multicolumn{5}{|c|}{ 1. Características personales y profesionales: edad, años } \\
\hline & Total & Tutoras & Tutores $\mathrm{C}$ & Grado de significación \\
\hline Media & 46.3 & 45.5 & 47.8 & 0.000 \\
\hline DT & 8.6 & 8.6 & 8.5 & \\
\hline \multicolumn{5}{|c|}{ 2. Características personales y profesionales: género \% } \\
\hline \multicolumn{3}{|c|}{ Tutores } & \multicolumn{2}{|c|}{65.0} \\
\hline \multicolumn{3}{|c|}{ Tutoras } & \multicolumn{2}{|r|}{35.0} \\
\hline \multicolumn{5}{|c|}{ 3. Características personales y profesionales: experiencia docente, años } \\
\hline & Total & Tutoras & Tutores $\mathrm{C}$ & Grado de significación \\
\hline Media & 19.2 & 18.5 & 20.5 & 0.001 \\
\hline DT & 9.9 & 9.9 & 10.2 & \\
\hline \multicolumn{5}{|c|}{ 4. Características personales y profesionales: experiencia como tutor/a, años } \\
\hline & Total & Tutoras & Tutores $\mathrm{C}$ & Grado de significación \\
\hline Media & 12.5 & 12.5 & 12.5 & --- \\
\hline DT & 8.9 & 8.8 & 9.0 & \\
\hline \multicolumn{5}{|c|}{ 5. Características personales y profesionales: según titularidad del centro \% } \\
\hline & & Público & Privado-concertado & o Privado \\
\hline 1. Total & & 81.6 & 17.4 & 1.0 \\
\hline 2. Tutoras & & 81.8 & 17.4 & 0.8 \\
\hline 3. Tutores & & 81.1 & 17.6 & 1.3 \\
\hline \multicolumn{5}{|c|}{ 6. Características personales y profesionales: según titularidad del centro y género \% } \\
\hline & & Público & Privado-concertado & O Privado \\
\hline 1. Tutoras & & 65.2 & 64.7 & 53.8 \\
\hline 2. Tutores & & 34.8 & 35.3 & 46.2 \\
\hline Total & & 100.0 & 100.0 & 100.0 \\
\hline \multicolumn{5}{|c|}{$\begin{array}{l}\text { 7. Características personales y profesionales: número de horas semanales que se dedican a } \\
\text { la función tutorial, según género. Media, horas. }\end{array}$} \\
\hline & Total & Tutoras & Tutores $\mathrm{C}$ & Grado de significación \\
\hline Media & 4.9 & 5.1 & 4.5 & 0.000 \\
\hline DT & 2.2 & 2.2 & 2.1 & \\
\hline
\end{tabular}




\section{Variables e Instrumentos}

Con la prueba que hemos elaborado y puesto a disposición de tutores y tutoras consultados pretendemos realizar un estudio exploratorio que nos informe sobre la actual situación de la función tutorial en las enseñanzas y etapas indicadas. Con ese objeto hemos analizado la validez de contenido mediante "juicio de expertos", cinco expertos han analizado las primeras propuestas del protocolo (Cuervo-Martínez y Escobar-Pérez, 2008). Respecto a la validez del constructo podemos señalar que la medida de adecuación muestral de Kaiser-Meyer-Olkin (KMO) tiene un valor de 0.951 y la prueba de esfericidad de Bartlett tiene un nivel de significación de 0.000. En el análisis factorial el método de extracción utilizado ha sido el Análisis de Componentes Principales y el método de rotación Varimax con Kaiser. La rotación ha convergido en 6 iteraciones. Seis factores explican el $72.3 \%$ de la varianza.

Por otro lado, las funciones tutoriales que hemos propuesto para valorar por tutores/as se corresponden con lo señalado en el artículo 91 de de la Ley Orgánica de 2/2006, de 3 de Mayo de Educación.

El coeficiente de de fiabilidad de Cronbach de la prueba tiene un valor de Alpha $=0,95$ (en la escala de 0 a 1) en el total de los 44 elementos analizados. Tres de ellos referidos a la valoración de la formación inicial y permanente tienen un coeficiente de Cronbach de 0.77. En temas de formación permanente su coeficiente es 0.93. La satisfacción laboral, importancia del tutor/a en el funcionamiento del centro y en la enseñanza del alumnado, alcanzan un coeficiente de fiabilidad de 0.76. El de la importancia de las funciones de tutor/a es de 0.89. Atención y dedicación a distintas funciones de tutor/a tienen un coeficiente de Cronbach 0.98. Respecto al reconocimiento y colaboración en su función, la carrera profesional, retribución económica y méritos, alcanzan un coeficiente de 0.95 .

Para realizar los análisis estadísticos correspondientes recurrimos a los descriptivos, diferencia de medias y análisis multivariado (utilizando el método Scheffe).

Tabla 2. Coeficientes de fiabilidad de Cronbach

\begin{tabular}{lc}
\hline \multicolumn{1}{c}{ Temas } & $\begin{array}{c}\text { Coeficiente de } \\
\text { Cronbach }\end{array}$ \\
\hline 1. Valoración de la formación inicial y permanente (3 elemento) & 0.77 \\
\hline 2. Temas de formación permanente (13 elementos) & 0.93 \\
\hline $\begin{array}{l}\text { 3. Satisfacción. Importancia del tutor/a en el funcionamiento del centro y } \\
\text { en la enseñanza del alumnado (3 elementos) }\end{array}$ & 0.76 \\
\hline 4. Importancia de las funciones de tutor/a (8 elementos) & 0.89 \\
\hline $\begin{array}{l}\text { 5. Atención y dedicación a distintas funciones del tutor/a (8 elementos) } \\
\text { 6. Reconocimiento y colaboración. Carrera profesional, retribución eco- } \\
\text { nómica, méritos (9 elementos) }\end{array}$ & 0.98 \\
\hline Total (44 elementos) & 0.95 \\
\hline
\end{tabular}


La prueba consta de elementos referidos a: 1. Variables de clasificación (género, edad, tiempo de experiencia profesional, tiempo de experiencia como tutor/a, titularidad del centro, enseñanzas y/o etapas, número alumnos/as del centro y número horas semanales dedicadas a la tutoría). 2. Formación inicial y permanente. 3. Temas de formación permanente (normativa, pedagogía-psicología, nuevas tecnologías, resolución de conflictos y convivencia, gestión del cambio e innovación, trabajo cooperativo, metodología didáctica, atención a las familias, dinámica de grupos, organización y gestión de equipos docentes, orientación escolar, evaluación del rendimiento académico y habilidades sociales). 4. Satisfacción laboral. 5. Importancia del tutor/a en el funcionamiento del centro y en la enseñanza del alumnado. 6. Reconocimiento y colaboración de tutores/as en sus tareas. 7. Carrera profesional, retribución económica y méritos. 8. Importancia de las funciones de tutor/a. 9. Atención y dedicación a distintas funciones de tutor/a.

\section{Resultados}

\section{Formación inicial y permanente}

Tutores/as valoran (en la escala de 0 a 10 puntos, siendo 0 "nada adecuada" y 10 "muy adecuada") con tan sólo 2.8 puntos la formación inicial, respecto a la función tutorial, recibida en sus estudios universitarios y la que reciben los actuales estudiantes de profesorado con 4.1 puntos en la escala indicada. A la formación permanente, respecto a sus funciones, impartida por parte de las administraciones educativas le otorgan una valoración media de 3.9 puntos. En ninguno de los tres aspectos estudiados se establecen diferencias significativas (nivel de significación 0.05) entre las valoraciones que se otorgan en función del género.

Tabla 3. Formación inicial y permanente

\begin{tabular}{|c|c|c|c|}
\hline \multicolumn{4}{|c|}{ Escala de 0 a 10 puntos. siendo 0 "nula o nada adecuada" y el 10 "muy adecuada } \\
\hline \multicolumn{4}{|c|}{ 1. Formación inicial, respecto a la función tutorial, recibida en sus estudios universitarios } \\
\hline & Total & Tutoras & Tutores \\
\hline Media & 2.8 & 2.9 & 2.8 \\
\hline DT & 2.6 & 2.6 & 2.6 \\
\hline Grado de significación & & ----- & \\
\hline \multicolumn{4}{|c|}{$\begin{array}{l}\text { 2. Formación que reciben los actuales estudiantes de profesorado respecto a la función } \\
\text { tutorial }\end{array}$} \\
\hline & Total & Tutoras & Tutores \\
\hline Media & 4.1 & 4.1 & 4.0 \\
\hline DT & 2.3 & 2.3 & 2.2 \\
\hline Grado de significación & & ---- & \\
\hline \multicolumn{4}{|c|}{$\begin{array}{l}\text { 3. Formación permanente que reciben tutores y tutoras por parte de las administraciones } \\
\text { educativas }\end{array}$} \\
\hline & Total & Tutoras & Tutores \\
\hline Media & 3.9 & 4.0 & 3.8 \\
\hline DT & 2.5 & 2.5 & 2.4 \\
\hline Grado de significación & & --- & \\
\hline
\end{tabular}




\section{Importancia de diferentes temas para la formación continua como tutor/a}

Tutores/as consideran importantes para su formación permanente, en su función tutorial, trece temas propuesto, (valorados en una escala de 0 a 10 puntos, siendo el 0 "nada importante" y el 10 "muy importante"). Valoraciones que tienen un recorrido de 2.3 puntos, siendo el menor valor, de 6.6 puntos, la "normativa" y el mayor, de 8.9 puntos, la "resolución de conflictos y convivencia". Entre siete y ocho puntos se valoran "nuevas tecnologías" (7.1), "gestión del cambio e innovación" (7.5), "metodología didáctica" (7.9) y "organización y gestión de equipos docentes" (7.6). Los restantes temas tienen una valoración entre ocho y nueve puntos, "pedagogía-psicología" (8.3), "trabajo cooperativo" (8.1), "atención a las familias" (8.6), dinámica de grupos (8.3), "orientación escolar" (8.2), "evaluación del rendimiento escolar" (8.0) y "habilidades sociales" (8.6).

Tabla 4. Formación permanente de tutores y tutora

\begin{tabular}{|c|c|c|c|}
\hline \multicolumn{4}{|c|}{ Escala de 0 a 10 puntos. siendo 0 "nula o nada adecuada" y el 10 "muy adecuada } \\
\hline \multicolumn{4}{|c|}{ 1. Normativa } \\
\hline & Total & Tutoras & Tutores \\
\hline Media & 6.6 & 6.7 & 6.5 \\
\hline DT & 2.3 & 2.3 & 2.2 \\
\hline Grado de significación & & ----- & \\
\hline \multicolumn{4}{|c|}{ 2. Pedagogía-psicología } \\
\hline & Total & Tutoras & Tutores \\
\hline Media & 8.3 & 8.5 & 7.9 \\
\hline DT & 2.0 & 2.0 & 2.1 \\
\hline Grado de significación & & 0.000 & \\
\hline \multicolumn{4}{|c|}{ 3. Nuevas tecnologías } \\
\hline & Total & Tutoras & Tutores \\
\hline Media & 7.1 & 7.2 & 6.9 \\
\hline DT & 2.0 & 2.0 & 2.1 \\
\hline Grado de significación & & 0.002 & \\
\hline \multicolumn{4}{|c|}{ 4. Resolución de conflictos y convivencia } \\
\hline & Total & Tutoras & Tutores \\
\hline Media & 8.9 & 9.1 & 8.6 \\
\hline DT & 1.7 & 1.5 & 1.9 \\
\hline Grado de significación & & 0.000 & \\
\hline \multicolumn{4}{|c|}{ 5. Gestión del cambio e innovación } \\
\hline & Total & Tutoras & Tutores \\
\hline Media & 7.5 & 7.7 & 7.2 \\
\hline DT & 2.0 & 1.9 & 2.1 \\
\hline Grado de significación & & 0.000 & \\
\hline \multicolumn{4}{|c|}{ 6. Trabajo cooperativo } \\
\hline & Total & Tutoras & Tutores \\
\hline Media & 8.1 & 8.4 & 7.6 \\
\hline DT & 1.9 & 1.8 & 2.1 \\
\hline
\end{tabular}


Escala de 0 a 10 puntos. siendo 0 "nula o nada adecuada" y el 10 "muy adecuada Grado de significación 0.000

\begin{tabular}{|c|c|c|c|}
\hline \multicolumn{4}{|c|}{ 7. Metodología didáctica } \\
\hline & Total & Tutoras & Tutores \\
\hline Media & 7.9 & 8.2 & 7.5 \\
\hline DT & 2.0 & 1.9 & 2.1 \\
\hline Grado de significación & & 0.000 & \\
\hline \multicolumn{4}{|c|}{ 8. Atención a las familias } \\
\hline & Total & Tutoras & Tutores \\
\hline Media & 8.6 & 8.8 & 8.2 \\
\hline DT & 1.8 & 1.7 & 1.9 \\
\hline Grado de significación & & 0.000 & \\
\hline \multicolumn{4}{|c|}{ 9. Dinámica de grupos } \\
\hline & Total & Tutoras & Tutores \\
\hline Media & 8.3 & 8.5 & 8.0 \\
\hline DT & 1.8 & 1.7 & 1.9 \\
\hline Grado de significación & & 0.000 & \\
\hline \multicolumn{4}{|c|}{10. Organización y gestión de equipos docentes } \\
\hline & Total & Tutoras & Tutores \\
\hline Media & 7.6 & 7.8 & 7.3 \\
\hline DT & 2.0 & 1.9 & 2.2 \\
\hline Grado de significación & & 0.000 & \\
\hline \multicolumn{4}{|c|}{ 11. Orientación escolar } \\
\hline & Total & Tutoras & Tutores \\
\hline Media & 8.2 & 8.3 & 7.9 \\
\hline DT & 1.8 & 1.7 & 2.0 \\
\hline Grado de significación & & 0.000 & \\
\hline \multicolumn{4}{|c|}{ 12. Evaluación del rendimiento escolar } \\
\hline & Total & Tutoras & Tutores \\
\hline Media & 8.0 & 8.1 & 7.7 \\
\hline DT & 1.8 & 1.7 & 1.9 \\
\hline Grado de significación & & 0.000 & \\
\hline \multicolumn{4}{|c|}{ 13. Habilidades sociales } \\
\hline & Total & Tutoras & Tutores \\
\hline Media & 8.6 & 8.8 & 8.3 \\
\hline DT & 1.7 & 1.5 & 1.9 \\
\hline Grado de significación & & 0.000 & \\
\hline
\end{tabular}

\section{Importancia de diferentes temas para la formación permanente en función del género y la titularidad del centro}

En relación con la importancia que tienen para la formación permanente de tutores/as los temas que hemos propuesto, en función del género y de la titularidad del centro, (en una escala de 0 a 10 puntos, siendo 0 "ninguna importancia" y 10 "mucha importancia") observamos lo siguiente: 
En general, como ya hemos señalado, las tutoras valoran significativamente como más importantes para su formación que los tutores, todos los aspectos señalados (con excepción de la "normativa" en la que no se encuentran diferencias)

Las tutoras de los centros públicos dan significativamente más importancia, para su formación, a todos los aspectos propuestos (con excepción de la "normativa" y "nuevas tecnologías" en los que no se establecen diferencias). Por otro lado, las tutoras de centros privados-concertados también consideran significativamente más importante los aspectos indicados, con excepción de "normativa", "pedagogía-psicología" y "resolución de conflictos y convivencia".

Tabla 5. Formación permanente de tutores y tutoras, según género y titularidad del centro

Escala de 0 a 10 puntos. siendo 0 "nula o nada adecuada" y el 10 "muy adecuada 1. Valoración de la "normativa", según género y titularidad del centro. Media

\begin{tabular}{lccc}
\hline & Total & Público & Privado concertado \\
\hline 1. Total & 6.6 & 6.6 & 7.1 \\
\hline 2. Tutoras & 6.7 & 6.6 & 7.2 \\
\hline 3. Tutores & 6.5 & 6.4 & 6.9 \\
\hline Grado de significación & --- & --- & --- \\
\hline
\end{tabular}

2. Valoración de "pedagogía-psicología", según género y titularidad del centro. Media

\begin{tabular}{lccc}
\hline & Total & Público & Privado concertado \\
\hline 1. Total & 8.3 & 8.2 & 8.8 \\
\hline 2. Tutoras & 8.5 & 8.5 & 8.8 \\
\hline 3. Tutores & 7.9 & 7.8 & 8.7 \\
\hline Grado de significación & 0.000 & 0.000 & --- \\
\hline
\end{tabular}

3. Valoración de "nuevas tecnología", según género y titularidad del centro. Media

\begin{tabular}{lccc}
\hline & Total & Público & Privado concertado \\
\hline 1. Total & 7.1 & 7.0 & 7.6 \\
\hline 2. Tutoras & 7.2 & 7.1 & 7.8 \\
\hline 3. Tutores & 6.9 & 6.7 & 7.3 \\
\hline Grado de significación & 0.002 & --- & 0.003 \\
\hline
\end{tabular}

4. Valoración de "resolución de conflictos y convivencia", según género y titularidad del centro. Media

\begin{tabular}{lccc}
\hline & Total & Público & Privado concertado \\
\hline 1. Total & 8.9 & 8.9 & 9.2 \\
\hline 2. Tutoras & 9.1 & 9.1 & 9.3 \\
\hline 3. Tutores & 8.6 & 8.5 & 9.1 \\
\hline Grado de significación & 0.000 & 0.000 & --- \\
\hline
\end{tabular}

5. Valoración de "gestión del cambio e innovación", según género y titularidad del centro. Media

\begin{tabular}{lccc}
\hline & Total & Público & Privado concertado \\
\hline 1. Total & 7.5 & 7.4 & 8.0 \\
\hline
\end{tabular}


Escala de 0 a 10 puntos. siendo 0 "nula o nada adecuada" y el 10 "muy adecuada

\begin{tabular}{lccc}
\hline 2. Tutoras & 7.7 & 7.6 & 8.3 \\
\hline 3. Tutores & 7.2 & 7.1 & 7.5 \\
\hline Grado de significación & 0.000 & 0.001 & 0.001 \\
\hline
\end{tabular}

\begin{tabular}{lccc}
\hline \multicolumn{4}{c}{ 6. Valoración de "trabajo cooperativo", según género y titularidad del centro. Media } \\
\hline & Total & Público & Privado concertado \\
\hline 1. Total & 8.1 & 8.0 & 8.4 \\
\hline 2. Tutoras & 8.4 & 8.3 & 8.7 \\
\hline 3. Tutores & 7.6 & 7.5 & 7.9 \\
\hline Grado de significación & 0.000 & 0.000 & 0.001 \\
\hline
\end{tabular}

7. Valoración de "metodología didáctica", según género y titularidad del centro. Media

\begin{tabular}{lccc}
\hline & Total & Público & Privado concertado \\
\hline 1. Total & 7.9 & 7.9 & 8.3 \\
\hline 2. Tutoras & 8.2 & 8.1 & 8.6 \\
\hline 3. Tutores & 7.5 & 7.5 & 7.7 \\
\hline Grado de significación & 0.000 & 0.000 & 0.000 \\
\hline
\end{tabular}

8. Valoración de "atención a las familias", según género y titularidad del centro. Media

\begin{tabular}{lccc}
\hline & Total & Público & Privado concertado \\
\hline 1. Total & 8.6 & 8.7 & 8.9 \\
\hline 2. Tutoras & 8.8 & 8.5 & 9.1 \\
\hline 3. Tutores & 8.2 & 8.2 & 8.6 \\
\hline Grado de significación & 0.000 & 0.000 & 0.009 \\
\hline
\end{tabular}

9. Valoración de "dinámica de grupos", según género y titularidad del centro. Media

\begin{tabular}{lccc}
\hline & Total & Público & Privado concertado \\
\hline 1. Total & 8.3 & 8.3 & 8.5 \\
\hline 2. Tutoras & 8.5 & 8.4 & 8.7 \\
\hline 3. Tutores & 8.0 & 8.0 & 8.2 \\
\hline Grado de significación & 0.000 & 0.000 & 0.025 \\
\hline
\end{tabular}

10. Valoración de "organización y gestión de equipos docentes", según género y titularidad del centro. Media

\begin{tabular}{lccc}
\hline & Total & Público & Privado concertado \\
\hline 1. Total & 7.6 & 7.6 & 7.8 \\
\hline 2. Tutoras & 7.8 & 7.7 & 8.1 \\
\hline 3. Tutores & 7.3 & 7.3 & 7.3 \\
\hline Grado de significación & 0.000 & 0.002 & 0.001 \\
\hline
\end{tabular}

11. Valoración de "orientación escolar", según género y titularidad del centro. Media

\begin{tabular}{lccc}
\hline & Total & Público & Privado concertado \\
\hline 1. Total & 8.2 & 8.1 & 8.3 \\
\hline 2. Tutoras & 8.3 & 8.3 & 8.5 \\
\hline 3. Tutores & 7.9 & 7.9 & 8.0 \\
\hline Grado de significación & 0.000 & 0.001 & 0.025 \\
\hline
\end{tabular}

12. Valoración de "evaluación del rendimiento escolar", según género y titularidad del centro. Media

\begin{tabular}{lccc}
\hline & Total & Público & Privado concertado \\
\hline 1. Total & 8.0 & 7.9 & 8.3 \\
\hline
\end{tabular}


Escala de 0 a 10 puntos. siendo 0 "nula o nada adecuada" y el 10 "muy adecuada

\begin{tabular}{lccc}
\hline 2. Tutoras & 8.1 & 8.0 & 8.5 \\
\hline 3. Tutores & 7.7 & 7.7 & 7.8 \\
\hline Grado de significación & 0.000 & 0.003 & 0.001 \\
\hline \multicolumn{4}{c}{ 13. Valoración de “habilidades sociales", según género y titularidad del centro. Media } \\
\hline & Total & Público & Privado concertado \\
\hline 1. Total & 8.6 & 8.6 & 8.8 \\
\hline 2. Tutoras & 8.8 & 8.8 & 9.0 \\
\hline 3. Tutores & 8.3 & 8.2 & 8.4 \\
\hline Grado de significación & 0.000 & 0.000 & 0.008 \\
\hline
\end{tabular}

\section{Satisfacción, importancia de la tutoría y apoyo de las administraciones}

Tutores/as tienen una notable satisfacción en el desarrollo de su función tutorial. La valoran con 7.2 puntos en la escala de 0 a 10 puntos (siendo el valor 0 "ninguna satisfacción" y el 10 la "máxima satisfacción"). Consideran que tutores y tutoras son muy importantes para el buen funcionamiento del centro y para la calidad de la enseñanza que éste ofrece, con valoraciones respectivas de 8.4 y 8.6 puntos. Siendo mejor valorados por las tutoras (gs 0.000).

Tabla 6. Satisfacción e importancia de la función tutorial para el centro y la calidad de la enseñanza que ofrece a su alumnado

\begin{tabular}{lccc}
\hline \multicolumn{4}{c}{ Escala de 0 a 10. Siendo el valor 0 “ninguna satisfacción" y el 10 la "máxima satisfacción" } \\
\hline \multicolumn{4}{c}{ 1. Satisfacción en su cargo como tutor o tutora } \\
\hline Total & 7.2 & Tutoras & Tutores \\
\hline 2. DT & 2.0 & 7.4 & 6.9 \\
\hline Grado de significación & & 1.9 & 2.1 \\
\hline
\end{tabular}

Escala de 0 a 10. Siendo el valor 0 "nada importante" y el 10 "muy importante"

2. Importancia de la función tutorial que desarrolla para el buen funcionamiento del centro

\begin{tabular}{lccc}
\hline & Total & Tutoras & Tutores \\
\hline 1. Media & 8.4 & 8.6 & 8.1 \\
\hline 2. DT & 1.5 & 1.4 & 1.6 \\
\hline Grado de significación & & 0.000 & \\
\hline
\end{tabular}

3. Importancia de la función tutorial que desarrolla para la calidad de la enseñanza que ofrece el centro

\begin{tabular}{lccc}
\hline & Total & Tutoras & Tutores \\
\hline 1. Media & 8.6 & 8.8 & 8.3 \\
\hline 2. DT & 1.4 & 1.2 & 1.5 \\
\hline Grado de significación & & 0.000 & \\
\hline
\end{tabular}




\section{Reconocimiento del trabajo de tutores/as por parte de las administraciones educativas, profesorado del centro y familias}

Tutores/as dan una baja valoración, 3.0 puntos, (en un escala de 0 a 10, siendo el valor 0 "ningún reconocimiento" y el 10 "máximo reconocimiento") al reconocimiento que les otorgan las administraciones educativas en su función tutorial. No se encuentran diferencias significativas (nivel de significación 0.05) según el género. Las valoraciones que dan al profesorado del centro y a las familias respecto a dicho reconocimiento son 5.5 y 5.7 puntos, respectivamente. En ambos casos las tutoras otorgan una valoración significativamente mayor.

Tabla 7. Reconocimiento del trabajo de tutores y tutoras por parte de las administraciones educativas, profesorado del centro y familias

\begin{tabular}{|c|c|c|c|}
\hline \multicolumn{4}{|c|}{ Escala de 0 a 10. Siendo el valor 0 "ningún reconocimiento" y el 10 la "máximo reconocimiento" } \\
\hline \multicolumn{4}{|c|}{ 1. Reconocimiento del trabajo del tutor/a por parte de las administraciones educativas } \\
\hline & Total & Tutoras & Tutores \\
\hline 1. Media & 3.0 & 3.0 & 2.9 \\
\hline 2. DT & 2.8 & 2.8 & 2.8 \\
\hline Grado de significación & & --- & \\
\hline \multicolumn{4}{|c|}{ 2. Reconocimiento del trabajo del tutor/a por parte del profesorado del Centro } \\
\hline & Total & Tutoras & Tutores \\
\hline 1. Media & 5.5 & 5.7 & 5.1 \\
\hline 2. DT & 3.2 & 3.2 & 3.3 \\
\hline Grado de significación & & 0.003 & \\
\hline \multicolumn{4}{|c|}{ 3. Reconocimiento del trabajo del tutor/a por parte de las familias del Centro } \\
\hline & Total & Tutoras & Tutores \\
\hline 1. Media & 5.7 & 5.9 & 5.4 \\
\hline 2. DT & 3.2 & 3.2 & 3.3 \\
\hline Grado de significación & & 0.024 & \\
\hline
\end{tabular}

\section{Carrera profesional, retribución económica y colaboración con la función tutorial}

Respecto a si los tutores/as deben tener una carrera profesional en la que se tenga en cuenta desempeñar o haber desempeñado la función tutorial, se valora con 6.3 puntos (en una escala de 0 a 10, siendo 0 "nada de acuerdo" y 10 "muy de acuerdo"). Las tutoras valoran significativamente mejor la propuesta (gs 0.013).

Con 6.9 puntos valoran tutores/as que el desempeño de la función tutorial sea objeto de una retribución económica y que el ser o haber sido tutor/a sea tenido en cuenta en los concursos de méritos y de traslados en la función pública (y en su caso constituir mérito profesional en los centros privados). Valoración significativamente mejor la que realizan para ambas cuestiones las tutoras.

Por otro lado, tutores/as, consideran aceptable la colaboración que tienen en su tarea por parte del profesorado del equipo docente, de las familias y alumnado y del equipo directivo $(6.2,5.6$ y 6.2 puntos respectivamente). En estos casos también las tutoras valoran significativamente mejor dicho reconocimiento. 
Tabla 8. Carrera profesional, retribución económica y colaboración con la función directiva

Escala de 0 a 10. Siendo 0 "nada de acuerdo" y 10 "muy de acuerdo".

1. ¿Los docentes deberían tener una carrera profesional en la que se tenga en cuenta ser $o$ haber sido tutor/a?

\begin{tabular}{lccc}
\hline & Total & Tutoras & Tutores \\
\hline 1. Media & 6.3 & 6.5 & 5.9 \\
\hline 2. DT & 3.6 & 3.5 & 3.8 \\
\hline Grado de significación & & 0.013 & \\
\hline
\end{tabular}

2. ¿El desempeño de la función tutorial debería ser objeto de una retribución económica?

\begin{tabular}{lccc}
\hline & Total & Tutoras & Tutores \\
\hline 1. Media & 6.9 & 7.1 & 6.6 \\
\hline 2. DT & 3.8 & 3.7 & 4.0 \\
\hline Grado de significación & & 0.039 & \\
\hline
\end{tabular}

3. ¿El ser o haber sido tutor/a debería ser tenido en cuenta en los concursos de méritos y de traslados en la función pública (y en su caso constituir mérito profesional en los centros privados)?

\begin{tabular}{lccc}
\hline & Total & Tutoras & Tutores \\
\hline 1. Media & 6.9 & 7.1 & 6.5 \\
\hline 2. DT & 3.8 & 3.7 & 3.9 \\
\hline Grado de significación & & 0.005 & \\
\hline
\end{tabular}

4. ¿Cómo valora la colaboración del resto del profesorado del equipo docente con su tarea tutorial?

\begin{tabular}{lccc}
\hline & Total & Tutoras & Tutores \\
\hline 1. Media & 6.2 & 6.4 & 5.8 \\
\hline 2. DT & 3.3 & 3.3 & 3.4 \\
\hline Grado de significación & & 0.003 & \\
\hline
\end{tabular}

5. ¿Cómo valora la colaboración de las familias y/o los alumnos/as con su tarea tutorial?

\begin{tabular}{lccc}
\hline & Total & Tutoras & Tutores \\
\hline 1. Media & 5.6 & 5.8 & 5.2 \\
\hline 2. DT & 3.1 & 3.1 & 3.2 \\
\hline Grado de significación & & 0.001 & \\
\hline
\end{tabular}

6. ¿Cómo valora la colaboración del equipo directivo con su tarea tutorial?

\begin{tabular}{lccc}
\hline & Total & Tutoras & Tutores \\
\hline 1. Media & 6.2 & 6.4 & 5.9 \\
\hline 2. DT & 3.5 & 3.4 & 3.7 \\
\hline Grado de significación & & 0.012 & \\
\hline
\end{tabular}

\section{Importancia de las funciones de tutor/a para la educación del alumnado y el funcionamiento del centro, según género}

Las valoraciones sobre la importancia que tutores/as otorgan a sus funciones en relación con la educación del alumnado y el funcionamiento del centro (en una escala de 0 a 10 puntos, siendo el 0 el "nada importante" y el 10 "muy importante") tienen un recorrido de 1.6 puntos. Siendo la mayor valoración la que recibe el aspec- 
to de "facilitar la integración del alumnado", con 9.1 puntos, y la menor la de "coordinar las actividades complementarias", con 7.5 puntos. En cualquier caso debemos tener en cuenta que la muestra de la que tenemos información corresponde a tutores/ as de las diferentes enseñanzas y etapas no universitarias y que en ellas no todas las funciones se desarrollan de igual manera. Las restantes funciones tienen valoraciones entre ocho y nueve puntos. Todas las funciones indicadas son significativamente mejor valoradas por las tutoras.

Tabla 9. Importancia de las funciones de tutor/a para la educación del alumnado y el funcionamiento del centro, según género

\begin{tabular}{|c|c|c|c|c|}
\hline \multicolumn{5}{|c|}{ Escala de 0 a 10 puntos. Siendo el 0 el "nada importante" y el 10 "muy importante" } \\
\hline & 1. Tutoras & 2. Tutores & Total & Gs \\
\hline $\begin{array}{l}\text { 1. Participar en la elaboración y desarrollo del plan de } \\
\text { acción tutorial y en las actividades de orientación }\end{array}$ & 8.1 & 7.6 & 7.9 & 0.000 \\
\hline $\begin{array}{l}\text { 2. Coordinar el proceso de evaluación de alumnos y } \\
\text { alumnas del grupo }\end{array}$ & 8.6 & 8.1 & 8.4 & 0.000 \\
\hline $\begin{array}{l}\text { 3. Coordinar el equipo docente en relación con el } \\
\text { alumnado del grupo }\end{array}$ & 8.6 & 8.2 & 8.5 & 0.000 \\
\hline $\begin{array}{l}\text { 4. Atender las dificultades de aprendizaje del alumna- } \\
\text { do de su tutoría }\end{array}$ & 8.8 & 8.2 & 8.6 & 0.000 \\
\hline $\begin{array}{l}\text { 5. Facilitar la integración del alumnado en el grupo y } \\
\text { fomentar su participación en las actividades del centro }\end{array}$ & 9.1 & 8.6 & 8.9 & 0.000 \\
\hline $\begin{array}{l}\text { 6. Orientar y asesorar a los alumnos y alumnas y/o a } \\
\text { las familias sobre sus posibilidades académicas y pro- } \\
\text { fesionales }\end{array}$ & 8.5 & 8.2 & 8.4 & 0.000 \\
\hline $\begin{array}{l}\text { 7. Coordinar las actividades complementarias del } \\
\text { alumnado del grupo }\end{array}$ & 7.5 & 6.9 & 7.3 & 0.000 \\
\hline $\begin{array}{l}\text { 8. Informar a las familias (o al alumnado en el caso de } \\
\text { mayoría de edad) sobre lo relacionado con las acti- } \\
\text { vidades docentes complementarias del alumnado del } \\
\text { grupo }\end{array}$ & 8.9 & 8.5 & 8.8 & 0.000 \\
\hline
\end{tabular}

\section{Atención y dedicación como tutor/a a las funciones de la acción tutorial, según género}

El recorrido de las valoraciones que tutores/as dan a la atención y dedicación que tienen respecto a las funciones de su acción tutorial, en la escala de 0 a 10 puntos (siendo el 0 el "ninguna atención y dedicación" y el 10 "la máxima atención y dedicación") es de 1.6 puntos. La menor valoración les corresponde a las funciones relativas a "participar en la elaboración y desarrollo del plan de acción tutorial y en las actividades de orientación" y "coordinar las actividades complementarias del alumnado del grupo", en ambos casos con 5.8 puntos. La mayor valoración la otorgan a la función de "informar a las familias (o al alumnado en el caso de mayoría de edad) sobre lo relacionado con las actividades docentes complementarias del alumnado del grupo" con 7.4 puntos. 
Las restantes funciones tienen las siguientes valoraciones: "coordinar el equipo docente en relación con el alumnado del grupo" y "orientar y asesorar a los alumnos y alumnas y/o a las familias sobre sus posibilidades académicas y profesionales", 6.8 puntos "atender las dificultades de aprendizaje del alumnado de su tutoría", 7.0 puntos, "coordinar el proceso de evaluación de alumnos y alumnas del grupo", 7.1 puntos y "facilitar la integración del alumnado en el grupo y fomentar su participación en las actividades del centro", 7.3 puntos. En todos los casos las tutoras consideran que prestan significativamente más atención y dedicación a dichas funciones que los tutores.

Tabla 10. Atención y dedicación como tutor o tutora a las funciones de la acción tutorial, según género

\begin{tabular}{|c|c|c|c|c|}
\hline \multicolumn{5}{|c|}{$\begin{array}{l}\text { Escala de } 0 \text { a } 10 \text { puntos. Siendo el } 0 \text { el "ninguna atención y dedicación" y el } 10 \text { "la } \\
\text { máxima atención y dedicación" }\end{array}$} \\
\hline & 1. Tutoras & 2. Tutores & Total & Gs \\
\hline $\begin{array}{l}\text { 1. Participar en la elaboración y desarrollo del plan de } \\
\text { acción tutorial y en las actividades de orientación }\end{array}$ & 5.8 & 5.1 & 5.6 & 0.001 \\
\hline $\begin{array}{l}\text { 2. Coordinar el proceso de evaluación de alumnos y } \\
\text { alumnas del grupo }\end{array}$ & 7.1 & 6.3 & 6.8 & 0.000 \\
\hline $\begin{array}{l}\text { 3. Coordinar el equipo docente en relación con el } \\
\text { alumnado del grupo }\end{array}$ & 6.8 & 6.0 & 6.3 & 0.000 \\
\hline $\begin{array}{l}\text { 4. Atender las dificultades de aprendizaje del alumna- } \\
\text { do de su tutoría }\end{array}$ & 7.0 & 6.2 & 6.7 & 0.000 \\
\hline $\begin{array}{l}\text { 5. Facilitar la integración del alumnado en el grupo y } \\
\text { fomentar su participación en las actividades del centro }\end{array}$ & 7.3 & 6.4 & 6.9 & 0.000 \\
\hline $\begin{array}{l}\text { 6. Orientar y asesorar a los alumnos y alumnas y/o a } \\
\text { las familias sobre sus posibilidades académicas y pro- } \\
\text { fesionales }\end{array}$ & 6.8 & 6.2 & 6.6 & 0.004 \\
\hline $\begin{array}{l}\text { 7. Coordinar las actividades complementarias del } \\
\text { alumnado del grupo }\end{array}$ & 5.8 & 5.2 & 5.6 & 0.002 \\
\hline $\begin{array}{l}\text { 8. Informar a las familias (o al alumnado en el caso de } \\
\text { mayoría de edad) sobre lo relacionado con las activida- } \\
\text { des docentes complementarias del alumnado del grupo }\end{array}$ & 7.4 & 6.6 & 7.1 & 0.000 \\
\hline
\end{tabular}

\section{DisCUSIÓN Y CONCLUSIONES}

Con el objetivo de conocer las valoraciones que tutores/as de centros de Educación Infantil, Educación Primaria, ESO, Bachillerato y FP realizan respecto a su formación, funciones y otros aspectos de la tutoría, hemos elaborado una prueba que ha sido cumplimentada por 1350 . El $64.9 \%$ son tutoras y el $35.1 \%$ restantes tutores, con una edad media de 46.3 años, experiencia docente 19.2 años y experiencia como tutor/a 12.5 años. Según la titularidad del centro el $81.6 \%$ ejerce su tutoría en centros públicos, el $17.4 \%$ en privados-concertados y el $1.0 \%$ en privados. Dedican a la acción tutorial una media semanal de 4.9 horas.

Dicha prueba consta de elementos referidos a: 1. Variables de clasificación. 2. Formación inicial y permanente. 3. Temas de formación permanente. 4. Satisfacción 
laboral, 5. Importancia del tutor/a en el funcionamiento del centro y en la enseñanza del alumnado. 6. Importancia de las funciones del tutor/a. 7. Atención y dedicación a distintas funciones. 8. Reconocimiento y colaboración. 9. Carrera profesional, retribución económica y méritos.

Tutores/as valoran con 2.8 puntos y 3.9 respectivamente (en la escala de 0 a 10) la formación que han recibido respecto a la función tutorial en su etapa universitaria y la que reciben en la actualidad por parte de las administraciones educativas. No se establecen diferencias significativas (nivel de significación 0.05) entre las valoraciones que otorgan en función del género. Valoraciones que coinciden con lo señalado por Cañas, Campoy y Pantoja (2005) y Rodríguez y Romero (2015), y que supone una necesaria revisión de la actual formación inicial y permanente que reciben profesores/as tutores/as.

Respecto a la importancia de diversos temas de su formación permanente observamos que las tutoras las valoran significativamente como más importantes que los tutores. Entendemos que las tutoras parecen presentar un mayor nivel de exigencia en su formación que sus colegas varones.

Las tutoras, por otra parte, muestran un mayor nivel de satisfacción en el desarrollo de su función tutorial que los tutores. El género es otro elemento distintivo respecto a la valoración que se da sobre la importancia de la tutoría para la calidad de la enseñanza y el buen funcionamiento del centro, en ambos casos las tutoras valoran significativamente mejor la importancia de la función tutorial. Respecto a la satisfacción del tutor/a en su tarea coincidimos con lo señalado por Anaya y López (2015); López (2013) y Serrano (2009). Satisfacción laboral que también para Anaya y López (2015) y Gil-Flores (2017) es mejor valorada por las tutoras.

Tutores y tutoras dan una baja valoración al reconocimiento que entienden les otorgan las administraciones educativas en su función tutorial, no encontrándose en este caso diferencias en función del género. Señalan al respecto López (2013) y Serrano (2009) la decepción que les supone la falta de reconocimiento y apoyo en su tarea. El reconocimiento que tienen por parte de profesorado y familias es bastante adecuado y también las tutoras le otorgan una mayor valoración. Parece que las administraciones educativas deben analizar sus actuaciones respecto al reconocimiento que le ofrecen al profesorado y de manea especial a tutores/as.

Las tutoras valoran mejor que en su carrera profesional se tenga en cuenta el haber sido o ser tutor/a, que dicha función debe ser objeto de una retribución económica y que sea tenido en cuenta en los concursos de méritos. También López (2013) y Serrano (2009) señalan que tutores/as reclaman una mejor retribución económica por su trabajo.

A tutores/as les parece aceptable la colaboración que tienen en su tarea por parte del equipo docente, de las familias y del equipo directivo. Las tutoras valoran significativamente mejor dicha colaboración. Por parte de Castro, Expósito, López y Navarro (2015) y Reparaz y Jiménez (2015) se considera que la relación y comunicación de las familias con el centro y de éste con aquella, es satisfactoria. 
Las valoraciones sobre la importancia que tutores/as otorgan a sus funciones para la educación del alumnado y el funcionamiento del centro podemos considerarlas como notables, siendo todas ellas significativamente mejor valoradas por las tutoras.

Aunque podemos considerar como adecuada la atención y dedicación que tutores/as manifiestan tener respecto a sus funciones tutoriales, son menos valoradas que las de su importancia respecto a las funciones de su acción tutorial. Las tutoras consideran que prestan una mejor atención y dedicación a su función tutorial que los tutores.

En relación con las diferencias en función del género de los diferentes aspectos que hemos analizado sobre la función tutorial podemos concluir que las tutoras tienen una mayor y mejor disposición para afrontar su formación permanente (en los temas que hemos señalado), un mayor nivel de satisfacción laboral y valoran mejor sus funciones para el funcionamiento del centro y la calidad de la educación que ofrece. Las tutoras también valoran mejor que se tenga en cuenta las funciones desarrolladas en la tutoría en su carrera profesional, en su retribución económica. También valoran mejor las tutoras la colaboración que le ofrecen el equipo docente, familias y equipo directivo. Otorgan, asimismo, una mejor valoración a la importancia de sus funciones tutoriales y su atención y dedicación para la educación del alumnado y el funcionamiento del centro.

Podemos señalar en definitiva, que en la mayor parte de los aspectos tutoriales analizados se encuentran diferencias de género, siendo las tutoras las que mejor los valoran. El análisis realizado nos indica que los perfiles profesionales de tutor y tutora presentan diferencias. Sería de interés analizar la incidencia que dichas diferencias pudieran suponer en la propia práctica docente y en la calidad de enseñanza (Álvarez, 2017; Cañas, Campy y Pantoja, 2005; López, 2013). ¿El trabajo de las tutoras supone una mejor práctica docente y mejor calidad de la enseñanza? Para analizar dichas cuestiones podríamos tener en cuenta las valoraciones al respecto de las familias (y el alumnado en su caso), directivos del centro y profesorado en general

\section{REFERENCIAS BIBLIOGRÁFICAS}

Álvarez, M. (2017). Hacia un modelo integrador de la tutoría en los diferentes niveles educativos. Revista Educatio Siglo XXI, 35(2), 21-42. http://doi. org/10.6018/j/298501.

Álvarez-Justel, J. (2017). La tutoría en secundaria. Revista Educatio Siglo XXI, 35(2), 65-90.

Anaya, D. y López, E. (2015). Satisfacción laboral del profesorado de educación secundaria. Revista de Investigación Educativa, 33(2), 435-452. http://doi.org/10.6018/ rie.33.2.202841.

Bermejo, M. L., Florencio, V., Ruiz, I. y Rabazo, M. J. (2014). Actitud del profesorado de primaria y secundaria en función del género ante los puntos fuertes de la integración. International Journal of Developmental and Educational Psychology, 3(1), 147-163. Recuperado de http://www.redalyc.org/pdf/3498/349851785016.pdf. 
Bolívar, A. (2007). La formación inicial del profesorado de secundaria y su identidad profesional. Estudios Sobre Educación, 12, 13-30.

Cañas, A., Campoy, T. J. y Pantoja, A. (2005). La función tutorial: valoración y necesidades del profesorado. Bordón: Revista de Pedagogía, 57(3), 297-214.

Castro, M., Expósito, E., Lizasoain, L., López, E. y Navarro, E. (2015). Acciones y actitudes diferenciales de los tutores y su relación con la participación de las familias. Participación Educativa, 4(7), 29-37.

Ceballos, N. (2017). La tutoría como espacio de democracia: una mirada desde la voz de los docentes en formación. Revista Española de Orientación y Psicopedagogía, 28(1), 86-103.

Cuervo-Martínez, A. y Escobar-Pérez, J. (2008). Validez de contenido y juicio de expertos: una aproximación a su utilización. Avances en Medición, 6, 27-36.

Esteve, J.M. (2007). La formación de profesores: bases teóricas para el desarrollo de programas de formación inicial. Revista de Educación, 350, 15-29.

Gil-Flores, J. (2017). Características personales y de los centros educativos en la explicación de la satisfacción laboral del profesorado. Revista de Psicodidáctica, 22(1), 16-22. http://doi.org/10.1016/S1136-1034(17)30039-4.

González-Palacios, A. y Avelino-Rubio, I. (2016). Tutoría: Una revisión conceptual. Revista de Educación y Desarrollo, 38, 57-68. Recuperado de http://www.cucs. udg.mx/revistas/edu_desarrollo/anterioresdetalle.php? $\mathrm{n}=38$.

Hernández, M. A., Viudez, N. y Guerrero, C. (2015). Percepción de las familias sobre las tutorías en la etapa de Educación Infantil. ENSAYOS, Revista de la Facultad de Educación de Albacete, 30(2). Recuperado de http://www.revista.uclm.es/index. php/ensayos.

Ley Orgánica de 2/2006, de 3 de mayo de Educación.

López, E. (2013). Aproximación a la percepción y satisfacción del profesor tutor de Secundaria Obligatoria respecto a su labor. Revista de Investigación en Educación, 11(1), 77-96. Recuperado de http://webs.uvigo.es/reined/.

Martín-Moreno, Q. (2008). El liderazgo femenino en los centros de los distintos niveles educativos. En J. Gairín y S. Antúnez (Eds.), Organizaciones educativas al servicio de la sociedad (pp. 188-199). Madrid: Wolters Kluwer.

Mateo, L. (2008). La Orientación y la Acción Tutorial en Educación Primaria. Revista digital para profesionales de la enseñanza, 10, 1-16. Recuperado de https://www. feandalucia.ccoo.es/docu/p5sd7451.pdf.

Quintanal, J. (2006). La función tutorial: presente y futuro. Educación y Futuro, 15, 9-23.

Reparaz, R. y Jiménez R. (2015). Padres, tutores y directores ante la participación de la familia en la escuela. Un análisis comparado. Participación Educativa, 4(7), 3945. Recuperado de https://sede.educacion.gob.es/publiventa/descarga.action?f_ codigo_agc=17380. 
Rodríguez, S. y Romero, L. (2015). La función tutorial en Educación Infantil y Primaria: desempeño profesional del profesorado. Revista Electrónica Interuniversitaria de Formación del Profesorado, 18(2), 43-55. http://doi.org/10.6018/reifop.18.2.219131.

Santana, L. E. y Feliciano, L. A. (2006): La construcción de la acción tutorial desde las coordenadas de la investigación colaborativa. Revista de Educación, 340, 943971.

Santos, M. A., Godás, A., Lorenzo, M. y Gómez-Fraguela, J. A. (2010). Eficacia y satisfacción laboral de los profesores no universitarios: revisión de un instrumento de medida. Revista Española de Pedagogía, 245, 151-170. Recuperado de http:// reunir.unir.net/handle/123456789/3868.

Sanz, J. R., Hernando, I. y Mula, J. M. (2015). La percepción del profesorado de Educación Secundaria de la Comunidad Valenciana acerca de sus conocimientos profesionales. Estudios sobre Educación, 29, 215-234. https://doi. org/10.15581/004.29.215-234.

Serrano, C. (2009) Acción tutorial y clima escolar en los centros educativos a juicio del profesorado de Educación Secundaria Obligatoria. Pulso, 32, 95-121.

Torres, J. A. (2010). Análisis del grado de satisfacción del profesorado de educación secundaria en el desarrollo de su labor docente. Contextos Educativos: Revista de Educación, 13, 27-42. http://doi.org/10.18172/con.624.

Valenzuela, C. y Sales, A. (2016). Los efectos de la participación dentro del aula ordinaria. Revista nacional e internacional de educación inclusiva, 9(2), 71-86. 